\title{
Reflexiones en torno a la evolución histórica del concepto de la educación a distancia
}

\section{Reflection concerning the historical evolution of the concept distance learning Reflexões sobre a evolução histórica do conceito de educação à distância}

\author{
Gilbert Ulloa Brenes \\ Universidad Estatal a Distancia \\ San José, Costa Rica. \\ gulloa@uned.ac.cr \\ (D) https://orcid.org/0000-0003-4138-2300
}

Recibido - Received - Recebido: 14 / 12 / 2020 Corregido - Revised - Revisado: 20 / 04 / 2021 Aceptado - Accepted - Aprovado: 13 / 05 / 2021

\author{
DOI: https://doi.org/10.22458/ie.v23i34.3364 \\ URL: https://revistas.uned.ac.cr/index.php/innovaciones/article/view/3364
}

\begin{abstract}
Resumen: En este ensayo se exponen algunos planteamientos en torno al devenir histórico del concepto y fundamentos de la educación a distancia, con el objetivo de indagar cómo se ha entendido la educación a distancia según diferentes teorías y desde su vínculo con las tecnologías de la comunicación. Para ello se efectuó una revisión bibliográfica y se seleccionaron textos de reconocidos autores por sus aportes a la reflexión acerca de la educación a distancia. Un hallazgo significativo de este ejercicio es que la construcción conceptual de la educación a distancia y, por ende, de sus fundamentos pedagógicos es reciente, aproximadamente hacia la década de 1960, a pesar de que se trata de una modalidad educativa que tiene sus antecedentes remotos en la invención de la comunicación escrita. De ahí que se concluya que la evolución histórica de esta modalidad educativa está asociada al devenir propio de las tecnologías de la comunicación y a la manera como estas permiten gestionar la distancia.
\end{abstract}

Palabras Clave: Educación, Educación a distancia, Tecnologías de la información, Pedagogía, Aprendizaje

Summary: This essay portraits some of the blueprints concerning the historical path of the concept and the foundations of distance learning aiming at bringing to light the concept's different theories and their link with communication technologies. Therefore, a bibliographical review was carried out to select texts by renown authors on the field that contributed to the reflection in regard to distance learning and their linked with communication technologies. A significant finding was to stumble upon the ideia that the conceptual construction of distance learning and hence its pedagogical foundations are recent approximately in the 1960's; in spite of it having its remote origins long before the invention of written communication. The conclusions lead to the conviction that the historical evolution of this education model is linked with the evolution of communication technologies themselves and the way in which they allow distance to be managed.

Keywords: distance learning, information technologies, pedagogy, learning

Resumo: Neste ensaio, algumas abordagens são expostas em torno da evolução histórica do conceito e dos fundamentos da educação à distância, com o objetivo de investigar como a educação à distância foi entendida de acordo com diferentes teorias e a partir de sua ligação com as tecnologias de comunicação. Para este fim, foi realizada uma revisão bibliográfica e foram selecionados textos de autores reconhecidos por suas contribuições para a reflexão sobre educação à distância. Uma descoberta significativa deste exercício é que a construção conceitual da educação à distância e, portanto, de seus fundamentos pedagógicos é recente, aproximadamente por volta dos anos 60, apesar de ser uma modalidade educacional que tem seus antecedentes remotos na invenção da comunicação escrita. Assim, conclui-se que a evolução histórica desta modalidade educativa está associada à evolução das tecnologias de comunicação e à forma como estas permitem a gestão da distância.

Palavras chave: educação, educação à distância, tecnologias da informação, pedagogia, aprendizagem 


\section{INTRODUCCIÓN}

La educación a distancia es hoy una de las modalidades más relevantes en educación, debido al creciente interés, como advierten Simonson, Smaldino \& Zvacek (2017), porque en diferentes escenarios educativos se faciliten formas de aprendizaje que trasciendan la presencia física en un salón de clase. Su auge, también se ha dado por el avance de las tecnologías de la comunicación y los enfoques que dan cuenta de sus implicaciones pedagógicas.

Sin embargo, cuando se trata de determinar lo más específicamente posible la diferencia entre la educación a distancia respecto de la presencial, lo común es que la reflexión concluya en aspectos como la presencia física, el uso de medios y la capacidad para cubrir las necesidades de un tipo particular de estudiantes que, por diversas razones, no pueden participar de procesos educativos que ameriten su presencia prolongada en un determinado espacio, todo ello a través de ciertos recursos técnicos.

Pero esa distinción entre presencia y distancia no resulta ya tan clara en el contexto de la pandemia provocada por el virus responsable de la COVID-19, cuando muchos de los procesos de educación presencial se convirtieron en estrategias de enseñanza remota en emergencia cuyo "... objetivo principal [...] no es recrear un ecosistema educativo robusto, sino más bien proporcionar acceso temporal a la instrucción y a los apoyos instructivos de una manera rápida y fácil de instalar durante una emergencia o crisis" (Hodges, Moore, Lockee, Trust y Bond, 2020, p. 17).

A pesar de que no se trate de la construcción de un ecosistema educativo robusto, la aplicación de tecnologías digitales en escenarios educativos que migraron, al menos provisionalmente, de la presencialidad a la enseñanza remota, trae consigo la necesaria revisión de lo que la dinámica presencia-distancia implica en este contexto, sobre todo si se considera que el uso de ese tipo de tecnologías tiene muy diferentes repercusiones pedagógicas, según las modalidades educativas se hayan concebido en sus orígenes para la presencialidad o a distancia (Giacomelli, 2019; Santos-Junior \& Monteiro, 2020).

Es decir, no basta con emplear herramientas digitales y virtualizar procesos educativos para convertir un sistema educativo tradicionalmente presencial, en uno a distancia. Esta última modalidad tiene tras de sí una amplia historia, marcada por teorías, modelos y experiencias que desde hace más de 70 años han coadyuvado en su configuración.

Así, la educación a distancia lejos de ser una respuesta de emergencia a coyunturas educativas adversas, es una modalidad educativa con características y fundamentos propios, los cuales hacia la segunda mitad del siglo XX han sido objeto de una reflexión pedagógica más sistemática.

En la línea de esas reflexiones, este ensayo tiene el propósito de indagar cómo se ha entendido la educación a distancia, según diferentes teorías y desde su vínculo con las tecnologías de la comunicación.

Para ello, la primera parte del desarrollo propone una breve mirada en torno a diferentes concepciones de la educación a distancia, para luego, en su segunda sección, discutir alrededor de las generaciones que han permitido comprender el devenir de esta modalidad educativa. Las reflexiones finales de este ensayo ofrecen una síntesis de tono más interpretativo, a partir de los argumentos que se esbozan a lo largo del recorrido teórico e histórico que se efectuó. 


\section{DESARROLLO}

\section{La modalidad de educación a distancia: dinámica de una concepción en perpetuo cambio}

Los más remotos orígenes de lo que hoy se conoce como educación a distancia se pueden ubicar desde la aparición de las primeras formas de escritura, paso fundamental dado por la humanidad para plasmar, más allá de la oralidad, experiencias y conocimientos que podían ser compartidos entre personas que no moraban en un espacio geográfico o un momento temporal común. Desde entonces, la educación en general y la que se desarrolla a distancia en particular, ha evolucionado de acuerdo con transformaciones tecnológicas que introducen a cada avance suyo formas más sofisticadas de interacción humana (Wedemeyer, 1981).

La educación a distancia por su insoluble vínculo con las tecnologías de la comunicación, y el devenir de estas a través de la historia, ha experimentado significativos cambios que se reflejan, primero en la interacción entre los sujetos que participan de ella, y en consecuencia, se reflejan luego en la forma de concebir a los sistemas educativos erigidos según esta modalidad educativa.

El tema en sí no es nuevo. Los primeros intentos por concebir qué es la educación a distancia tuvieron como principal referencia el papel de los medios de comunicación: desde las implicaciones educativas de la instrucción por correspondencia que emergió hacia mediados del siglo XIX, pasando por el debate planteado hacia 1983 por Richard Clark (citado por Kirkwood, 2013), para quien los medios eran solamente vehículos para almacenar o enviar información, pero cuyos efectos en el aprendizaje eran, aparentemente, nulos, hasta las críticas que Kozma (1991) dirigió contra Clark al formular que los medios tecnológicos si tienen un alto impacto en los procesos de aprendizaje.

Quizá el antecedente educativo que mejor se identifica como una de las primeras expresiones de la educación a distancia fue la compañía Pitman Training, la cual desde mediados del siglo XIX en Inglaterra inauguró una forma de instrucción vía correo postal, en la cual se capacitaba a personas interesadas en aprender taquigrafía. Más adelante, hacia 1878 el obispo John H. Vincent, funda el Chautauqua Literary and Scientific Circle, instancia con sede en Nueva York que ofrecía procesos educativos por correspondencia (Moore \& Kearsley, 2012).

Desde entonces, se popularizó la oferta de procesos instruccionales por correspondencia enfocados, principalmente, en la formación de competencias específicas para el desempeño de personas en labores técnicas requeridas por diferentes centros de trabajo de la época.

Sin embargo, fue a lo largo del siglo XX, sobre todo a partir de la década de 1960, con el aumento de la presión sobre los sistemas educativos presenciales, que mostraban grandes falencias para atender las necesidades educativas de sectores poblacionales crecientes y en evidente desigualdad respecto de las clases más favorecidas que sí accedían a los espacios de formación, cuando la educación a distancia emergió como una alternativa viable para democratizar el alcance de diferentes programas educativos (García Aretio, 1999); quizá hasta ese momento nunca se había pensado en la distancia como un factor pedagógico en general.

Desde entonces se han concebido diferentes definiciones de la modalidad denominada educación a distancia, la mayoría de las cuales enfatizan aspectos como: la no-presencialidad en la interacción docente-estudiante, el recurso a herramientas tecnológicas como vehículo medular del proceso de enseñanza-aprendizaje, la relevancia del individuo en su propia formación y la configuración de estilos de comunicación bidireccional (García Aretio, 1987). 
En específico, los primeros intentos por definir, de manera más precisa, qué era y qué implicaba educar a distancia estuvieron marcados por la necesidad de trascender la simplista denominación de "estudios por correspondencia", para abrir la discusión académica en torno a las implicaciones pedagógicas que se abrían con esta modalidad de estudios.

En ese sentido, se reconoce al pedagogo estadounidense Charles Wedemeyer como el precursor de esas primeras formulaciones conceptuales de la educación a distancia, a través de su noción de estudio independiente. En efecto, Wedemeyer (citado por Diehl \& Cano, 2019) sostenía una concepción de estudio independiente en la cual se recalcaba el papel del estudiantado:

... in which teachers and learners carry out their essential tasks and responsibilities apart from one another, communicating in a variety of ways, for the purposes of freeing internal learners from inappropriate class pacings or patterns, or providing external learners opportunity to continue learning in their own environments (p. 19).

Es decir, Wedemeyer ofrecía una perspectiva centrada no solo en el problema de la distancia física entre docentes y estudiantes como parte del aprendizaje, sino, además, en una visión quizá cercana a los postulados de la Escuela Nueva, preconiza una educación más acorde a las potencialidades y al contexto en que se desarrolla el sujeto aprendiente.

El acento un tanto individualista de esas primeras concepciones formuladas por Wedemeyer serían complementadas, más adelante, por la propuesta de teóricos como Börje Holmberg (1983; 1995; 2003), educador de origen sueco, quien destacaba ya hacia la década de 1960 la relevancia de los medios de comunicación electrónicos en el contacto entre docentes y aprendices, en buena medida retomó los aportes de Wedemeyer en torno al estudio independiente para dar un paso más e incluir la interacción comunicativa entre docentes y estudiantes en la conversación didáctica guiada.

Con esto, Holmberg (1983) apostaba por entender que la educación a distancia emergía como una modalidad particularmente óptima para aplicar formas de interacción que se podían ver como una conversación guiada en forma didáctica, la cual suponía que

... the character of good distance education resembles that of a guided conversation aiming at learning and that the presence of the typical traits of such a conversation facilitates learning. The distance-study course and the non-contiguous communication typical of distance education are seen as the instruments of a conversation-like interaction between the student on the one hand and the tutor counsellor of the supporting organization administering the study on the other. There is constant interaction ('conversation') between the supporting organization (authors, tutors, counsellors), simulated through the students' interaction with the pre-produced courses and real through the written and/or Telephone interaction with their tutors and counsellors (p. 114).

El tránsito desde una concepción del estudio independiente hacia esta perspectiva en la cual se enfatiza la conversación guiada como estrategia didáctica, representaría un viraje significativo apuntalado por las tecnologías de la comunicación disponibles para la época, en especial en el uso masivo de la telefonía fija; pues brindaba la oportunidad de una interacción docente-estudiante bastante más dinámica que la provista por la correspondencia.

Incluso, es plausible afirmar que esa noción de "conversación guiada" con fines didácticos elaborada por Holmberg, representa un elemento pedagógico que permanece vigente hasta hoy en la educación a distancia, amplificada gracias a las nuevas rutas de comunicación que ofrecen las tecnologías digitales y el uso intensivo de diferentes redes sociales, así como las perspectivas de ulteriores modelos 
pedagógicos como la del "diálogo mediado" propuesto por el reconocido pedagogo español Lorenzo García Aretio (2008; 2012).

Aún más, se trataría, mutatis mutandis, de una concepción precursora de las reflexiones que otros autores, como el argentino Mario Kaplún (1998) y su tesis de la educación comunicativa, esbozarían en relación con las repercusiones pedagógicas de la comunicación.

También hacia los años 60 del pasado siglo, el educador alemán Otto Peters postuló una visión "industrializada", la cual procuraba observar en la educación a distancia un proceso objetivo tendiente a una planificación estandarizada y racionalizada, sustentada en la tecnología como puntal de metas pedagógicas a gran escala, susceptibles entonces de masificación y, por ende, de ofrecerse como un producto comercial, en un modelo que apuntaba a emular la producción incluso en la era postindustrial (Peters, 1993; 2002).

Como era de esperar, el ascendiente neofordista de la industrialización educativa propuesta por Peters, desencadenó variadas críticas (Costa \& Aquino, 2011), sobre todo dirigidas a la aplicación acrítica en la educación de lógicas de producción pensadas para las grandes industrias y la maximización de sus procesos; no obstante, ha significado la revisión de una teoría que en criterio de diferentes autores, aún puede ofrecer un marco válido para comprender las implicaciones pedagógicas y económicas de la educación a distancia en la era de las sociedades del conocimiento (Zawacki-Richter, 2019).

Posterior a la década de 1970, se retoman las significativas transformaciones gestadas en lo pedagógico por las teorías cognitivas, Michael Grahame Moore formuló una perspectiva de la educación a distancia que evolucionó hacia la denominada teoría de la distancia transaccional, la cual enfatiza el rol del diálogo, la autonomía del estudiantado y la estructura de los diseños académicos como bases de la interacción (de ahí la noción de transacción entre sujetos) y fundamentos de la educación a distancia. El propio Moore (2019) explica que

Thus, the "transaction" in distance education is the interplay of the behaviors of teachers and learners in environments in which they are in separate places and have to communicate through a technology. It is this separation between learners and teachers that necessitates special "patterns of behavior" in how content and teaching are organized in courses and programs -that is their structure- and special "patterns of behavior" in how teachers interact with learners when using communications technologies in the tasks of creating knowledge -that is, through dialogue (p. 33).

Así, una efectiva interacción, apuntalada por el diálogo y reforzada desde una estructura curricular óptima permitirá que la distancia transaccional sea mínima. Además, Moore ha advertido que su teoría de la distancia transaccional se puede ver enriquecida con algunas más recientes formulaciones pedagógicas, como la noción de "enseñanza personalizada" y su énfasis en la autonomía del estudiantado, o el papel de las actuales tecnologías digitales al proporcionar nuevos escenarios para la promoción de interacciones dialógicas, que derivarían en estrategias como el denominado blended learning, o su versión más específica, el flipped classroom como estrategia didáctica, desde la cual la estructura curricular se abre a una más efectiva flexibilización de lo estructural-curricular.

Las voces críticas hacia la teoría de la distancia transaccional de Moore han reprochado el concepto de diálogo que ofrece; pues profundiza en términos de la interacción estudiante-estudiante en un grupo de aprendizaje (Gorsky \& Caspi, 2005; Reyes, 2013), o las falencias para dar cuenta, satisfactoriamente, de las implicaciones que tendría la comunicación sincrónica en la autonomía del estudiantado (Fallon, 2011).

Por otra parte, la teoría de la equivalencia postulada originalmente por Simonson y Schlosser hacia finales de la década de 1990, se puede entender como heredera de las teorizaciones sobre el aprendizaje 
individual que se remontan a autores como Wedemeyer y su propuesta del estudio independiente o las perspectivas en torno a la comunicación educativa en Holmberg.

En el caso de la teoría del estudio independiente de Wedemeyer, por ejemplo, coincide en el énfasis puesto en la adaptación de los procesos de aprendizaje a las condiciones individuales de cada estudiante, sobre todo a la adaptación de recursos pedagógicos y métodos que sean efectivos para que el estudiantado pueda aprender a su propio ritmo (Simonson, Zvacek \& Smaldino, 2017; Diehl \& Cano, 2019). Y en ese sentido, se trata de una teoría que coincidiría con la ya clásica definición de estudio independiente brindada por Moore (1977):

Independent Study is any educational programme in which the learning programme occurs separate in time and place from the teaching programme, and in which the learner has an influence at least equal to the teacher in determining goals, resources, and evaluation decisions (p. 11).

Sin embargo, resulta posible también hallar en ella vestigios de algunas de las ideas de la teoría de la interacción y la comunicación de Hölmberg, sobre todo a partir de la reformulación que este autor hiciera hacia mediados de la década de 1990, cuando advirtió de la relevancia que conlleva reconocer la heterogeneidad del estudiantado, la libertad de elección y la independencia con que podrían organizar sus estudios y el aprendizaje en profundidad que se puede alcanzar de manera individual (Simonson, Zvacek \& Smaldino, 2017; Diehl \& Cano, 2019).

Asimismo, otras perspectivas como la síntesis teórica ofrecida por Hilary Perraton, empatan con la perspectiva de la equivalencia, o al menos comparten una perspectiva pedagógica que puede denominarse pragmática, en tanto que se brinda un especial énfasis al uso de cualquier medio de aprendizaje en tanto este sirva para una comunicación y un aprendizaje efectivos (Simonson, Zvacek y Smaldino, 2017).

De forma sintética, la idea central de la teoría de la equivalencia consiste en que diferentes experiencias de aprendizaje se tornan equivalentes, si los resultados de aprendizaje que se consiguen en una u otra son los mismos y de similar calidad sin importar el entorno en el que se lleven a cabo.

La consecuencia de esta idea básica radica en que, para la teoría de la equivalencia, los aprendizajes del estudiantado a distancia deben ser equivalentes a los de cualquier otro estudiante de sistemas presenciales (local students). En otras palabras, deben proporcionarse experiencias de aprendizaje equivalentes, pero no idénticas, a cada estudiante, ya sea en un sistema presencial o a distancia. Por ello, se esperaría que el estudiantado, de acuerdo con sus características individuales, acceda a estrategias de instrucción diferenciadas pero equivalentes; es decir, se adapten a sus características pero que no impliquen un desigual acceso a los recursos pedagógicos y menos aún a una diferencia en la calidad. De ese modo, se garantizaría que "If the distance education course is effectively designed and equivalent experiences are available, then potential learners will reach the course's instructional objectives" (Simonson, Zvacek y Smaldino, 2017, p. 50).

La lógica de esta equivalencia deriva de la idea de que el estudiantado y el profesorado en un sistema a distancia no están presentes en un mismo espacio y pueden no realizar actividades sincrónicas, por lo cual el aprendizaje se efectúa por medios tecnológicos que han de permitir diferentes combinaciones de la ecuación lugar y tiempo.

Este repaso en torno a las diferentes perspectivas sobre la educación a distancia atestigua que la reflexión pedagógica alrededor de esta modalidad educativa ha cambiado en el tiempo, conforme los recursos tecnológicos ofrecen nuevas posibilidades para establecer procesos de aprendizaje que no se limitan a situaciones geográficas o temporales restringidas. 
Sin embargo, de acuerdo con la definición brindada por Schlosser \& Simonson (citados por Simonson, Smaldino \& Zvacek, 2017) quienes entienden esta como una modalidad educativa formal y fundamentada a nivel institucional "... where the learning group is separated, and where interactive telecommunications systems are used to connect learners, resources, and instructors" (p. 6), se puede afirmar que la educación a distancia ha estado históricamente compuesta por tres elementos clave: la relación que se establece entre docentes y estudiantes, la interactividad facilitada por las tecnologías de comunicación vigentes y las experiencias de aprendizaje que amalgaman el proceso educativo.

\section{Dinámicas tecnológicas y su impacto en la educación a distancia}

Como se ha mencionado en diferentes momentos de este ensayo, las concepciones en torno a la educación a distancia son constructos pedagógicos formulados a propósito de las interacciones que se pueden dar en esta modalidad, pero también son fruto del propio avance de las tecnologías de la comunicación que, en diferentes momentos, han propiciado determinadas formas de relación entre sujetos cuya distancia física deja de serlo en virtud de la cercanía que virtualmente ofrecen esas tecnologías.

Así, se puede identificar también distintas perspectivas en torno a las implicaciones pedagógicas de la educación a distancia a lo largo del tiempo, en la cual se enfatiza la dinámica dada entre sujetos, medios de comunicación y experiencias de aprendizaje en diferentes momentos históricos.

En ese sentido, de acuerdo con Moore \& Kearsley (2012, pp. 23-44), la evolución de la educación a distancia ha estado marcada por avances tecnológicos que traen aparejadas nuevas formas de entender la dinámica educativa en sí, con lo cual se perciben distintas "generaciones" dentro de esta modalidad educativa.

Cuando antes de la segunda mitad del siglo XX se sentaban las bases de la reflexión pedagógica alrededor de la educación a distancia, con perspectivas como la de Wedemeyer y su enfoque en el estudio independiente, se asistía a un entorno histórico que aún podía identificarse como parte de la primera generación de la educación a distancia, caracterizada por la comunicación vía correspondencia y se atisbaban resabios de una concepción meramente instruccional del aprendizaje, en la cual el vínculo entre docente y aprendiz tendía a la unidireccionalidad. En ese sentido, los aportes de Wedemeyer representaron un paso audaz al resaltar la autonomía del estudiante y la necesidad de maximizar sus capacidades, antes que limitarlo al papel de un receptor pasivo de contenidos transferidos vía correo postal.

Sin embargo, la comunicación posibilitada por los medios vigentes en esa época restringía, hasta cierto punto, que la interacción entre docente y estudiante fluyera de manera dialógica, de ahí que la autonomía estudiantil fuera puesta como base del andamio que permitiría a la persona discente ascender hasta los nuevos conocimientos.

La segunda generación, que puede datarse hacia la década de los 70 del pasado siglo, implicó una maximización de las comunicaciones, gracias a los avances tecnológicos que popularizaron el acceso a medios como la radio, la televisión, así como la diversificación de los medios impresos; todo ello redundó en una perspectiva desde la cual se atendió la actividad cognitiva del sujeto aprendiente, de paso, permitió una comunicación con matices más bidireccionales entre docentes y estudiantes.

Así, la distancia como brecha espacial y temporal tendía a estrecharse y propuestas como la conversación didácticamente guiada de Holmberg se mostraban particularmente fecundas, en un escenario histórico en el cual la comunicación sincrónica era ya una realidad.

Más adelante, la denominada tercera generación de la educación a distancia se caracterizó por la aparición, hacia finales de 1980, de las primeras computadoras, hito tecnológico que, aunado a la ya masiva 
difusión televisiva, propició una dinámica mucho más interactiva y comunicativa, que redundó en la configuración de tesis sobre la educación en general y la que se impartía a distancia en especial, sobre el rol activo tanto del docente como del discente en el aprendizaje. Con ello, los enfoques pedagógicos sustentados en el constructivismo avanzaron rápidamente y la comunicación como factor pedagógico ocupó un lugar más significativo en las reflexiones de diferentes especialistas involucrados en procesos educativos a distancia.

Y para finales de 1990 e inicios del siglo XXI, la llegada de la internet maximizó las posibilidades que desde al menos una década atrás ya se estaban fraguando, y con ello se pasó a la denominada cuarta generación de la educación a distancia, en la cual el estudiante, idealmente, no solo se torna constructor de conocimientos, sino además deviene intérprete activo de formas de aprendizaje que, mediadas por la figura docente, deben ser significativas. Se recalca, entonces, la triada que propuso Moore en su teoría de la distancia transaccional y en las posteriores reformulaciones hechas por otros autores: el diálogo como factor clave en la comunicación pedagógica, la estructura en el diseño de procesos educativos más versátiles y, en medio, la preponderancia de la autonomía como espacio para la acción del sujeto cognoscente.

Finalmente, la quinta denominada generación de la educación a distancia, que se gesta desde principios del siglo XXI hasta hoy, se muestra como un momento en el cual, quizá más que nunca, esta modalidad educativa se torna en una alternativa preponderante, al ofrecer la oportunidad de procesos pedagógicos más dinámicos, en los cuales se integran las nuevas tecnologías digitales con el ideal de sujetos cognoscentes interactivos, capaces incluso de producir y gestionar contenidos propios, y la persona docente se transformaría también en una especie de mediador-editor de los productos del estudiantado.

Dentro de este último escenario histórico, se incuba la aparición de la inteligencia artificial y quizá anuncie el advenimiento de una nueva generación, la sexta, en la educación a distancia. Lo que esa nueva fase augure para esta modalidad educativa está aún por verse, pero ciertamente tanto el rol de la persona docente como el del estudiantado se verán afectados por las condiciones pedagógicas que se logren articular en un nuevo escenario.

Además, como se anotó al principio, si un escenario de emergencia como el generado con la pandemia provocada por el virus responsable de la COVID-19 ha obligado a los sistemas educativos presenciales a migrar, de manera transitoria, hacia experiencias de educación remota, en ello se puede observar una tendencia que, quizá en el largo plazo, haga de la educación a distancia una vía más que alternativa para, a veces, los excesivamente tradicionales entornos de educación presencial.

Ese escenario conlleva, claro está, a nuevos retos y nuevas oportunidades no solo para la aplicación de modalidades educativas a distancia sino, además, para la siempre necesaria reflexión pedagógica que permita novedosas vías para concebir la educación a distancia y sus diferentes componentes, de cara a un futuro en el que los avances en las tecnologías de la comunicación permitan entender la distancia como una forma de cercanía pedagógica.

\section{SÍNTESIS Y REFLEXIONES FINALES}

Efectivamente, como se sugiere al final de la sección anterior, es tarea perentoria la búsqueda de nuevas reflexiones y análisis capaces de dar cuenta de las características particulares de la educación a distancia en el contexto de las nuevas herramientas tecnológicas y los más contemporáneos procesos de comunicación; pues no se debe perder de vista que este tipo de análisis supeditados a la innovación técnica, la mediación desde herramientas tecnológicas, puede resultar vacío sin una reflexión que inquiera por los fundamentos antropológicos, epistemológicos, en fin, filosóficos de la distancia en la educación. 
Ello implica que se deba avanzar sobre la reflexión profunda en torno a la forma en que la relación docentes y estudiantado consigue hacer efectiva la riqueza de los procesos educativos, en términos de una mediación comunicativa capaz de promover la interactividad dialógica, para parafrasear la propuesta de Moore, y permita consolidar el derecho a la educación desde la modalidad a distancia (Rama, 2019).

Como se ha propuesto en este ensayo, la evolución histórica de la educación a distancia ha estado fuertemente asociada al devenir propio de las tecnologías de la comunicación y, por ello, es una parte fundamental de su propia evolución identificar cómo esas tecnologías permiten gestionar la distancia como factor pedagógico.

Las diferentes teorías analizadas aquí emergieron en consecuencia con ese desarrollo tecnológico, y aunque podrían ser valoradas como fruto de un contexto histórico muy específico, no deja de ser referentes válidos para la reflexión pedagógica que hoy se requiere. Por ejemplo, las ideas seminales de tesis como la del aprendizaje independiente ¿no están presentes en propuestas como las teorías de la autorregulación y su influjo en procesos educativos a distancia? O la industrialización planteada por Peters, ¿acaso no sigue siendo un dilema siempre presente cuando se trata de gestionar la educación a distancia?

Como sea, la educación a distancia ha estado presente desde las primeras formas de educación, aunque la definición de sus características particulares es relativamente reciente, al identificarse las primeras formulaciones teóricas específicas hacia la década de 1950. Eso implica, por una parte, un espacio de reflexión conceptual abierto a las nuevas formulaciones que se fragüen al calor de los avances tecnológicos, pero sin dejar de lado la dimensión pedagógica que comporta.

En ese sentido, la configuración teórica de la educación a distancia deviene en una constante síntesis dialéctica entre los aportes conceptuales del pasado, y las exigencias que las nuevas formas de comunicación traen consigo, en el marco de interacciones pedagógicas que sin duda están marcadas por el uso de los diferentes medios digitales disponibles en los albores de esta segunda década del siglo XXI.

\section{REFERENCIAS}

Costa, A. \& Aquino, M. (2011). Industrialização do ensino e política de educação a distância. Ciência em Movimento. XIII(26):41-51.

Diehl, W. \& Cano, L. (2019). Charles A. Wedemeyer and the first theorists. In M. Moore. \& W. Diehl (Eds.). Handbook of Distance Education, pp. 19-31. New York, USA: Routledge.

Fallon, G. (2011). Making the Connection: Moore's Theory of Transactional Distance and Its Relevance to the use of a Virtual Classroom in Postgraduate Online Teacher Education. Journal of Research on Technology in Education. 43(3):187-209.

García Aretio, L. (1987). Hacia una definición de educación a distancia. Boletín informativo de la Asociación Iberoamericana de educación Superior a distancia. 4(18):1-4.

García Aretio, L. (1999). Historia de la Educación a Distancia. RIED. 2(1):8-27. https://doi.org/10.5944/ ried.2.1.2084

García Aretio, L. (2008). Diálogo didáctico mediado. Boletín Electrónico de Noticias de Educación a Distancia, junio. Madrid, España: Universidad Nacional de Educación a Distancia.

García Aretio, L. (2012). El diálogo didáctico mediado en educación a distancia. Contextos Universitarios Mediados. 34(12).

Giacomelli, J. (2019). Tecnologia e ensino a distância: implicações, possibilidades e limites (do aprender e do ensinar). (Dissertação de Mestrado em Educação nas Ciências para a obtenção do Título de Mestre em Educação nas Ciências). Universidade Regional do Noroeste do Estado do Rio Grande do Sul. 
Gorsky, P. \& Caspi, A. (2005). A critical analysis of Transactional Distance theory. The Quarterly Review of Distance Education. 6(1):I-11.

Hodges, C., Moore, S., Lockee, B., Trust, T. y Bond, A. (2020). La diferencia entre la enseñanza remota de emergencia y el aprendizaje en línea, en P. Neira, C. Rodríguez y J. Villanueva (comps.). Enseñanza remota de emergencia: textos para la discusión, pp. 12-22. Lima, Perú: Th eLearning Factor. Recuperado de http://www.educaccionperu.org/wp-content/uploads/2020/04/ Ensen\%CC\%83anza-Remota-de-Emergencia-Textos-para-la-discusio\%CC\%81n.pdf

Holmberg, B. (1983). Guided didactic conversation in distance education. In D. Sewart, D. Keegan \& B. Holmberg (Eds.). Distance education: International perspectives, pp. 114-122. London, England: Croom Helm.

Holmberg, B. (1995). The Sphere of Distance-Education Theory Revisited. ZIFF Papiere 98. Hagen, Germany: FernUniversität.

Holmberg, B. (2003). Distance Education in Essence. An overview of theory and practice in the early twentyfirst century. Oldenburg, Germany: Bibliotheks-und Informationssystem der Carl von Ossietzky Universität Oldenburg.

Kaplún, M. (1998). Una pedagogía de la comunicación. Madrid, España: Ediciones de la Torre.

Kirkwood, A. (2013). Media are "mere vehicles" - Under-substantiated claims from comparative studies. A review of Richard E. Clark (ed.) Learning from Media: Arguments, Analysis, and Evidence. Open Learning. 28(2):153-163.

Kozma, R. (1991). Learning With Media. Review of Educational Research. 61(2):179-211.

Kozma, R. (1994). Will media influence learning? Reframing the debate. Educational Technology Research and Development. 42(2):7-19.

Moore, M. (1977). On a Theory of Independent Study. Ziff Papiere. (16):1-27.

Moore, M. (2019). The Theory of Transactional Distance. In M. Moore. \& W. Diehl (Eds.). Handbook of Distance Education, pp. 32-46. New York, USA: Routledge.

Moore, M. \& Kearsley, G. (2012). Distance Education: A Systems View of Online Learning. California, USA: Wadsworth.

Peters, O. (1993). Distance education in a postindustrial society. In Keegan, D. (ed.). Theoretical principles of distance education, pp. 39-60. New York, USA: Routledge.

Peters, O. (2002). Distance education in transition. Developments and issues. Oldenburg, Germany: Bibliotheks-und Informationssystem der Carl von Ossietzky Universität Oldenburg.

Rama, C. (2019). Políticas, tensiones y tendencias de la Educación a Distancia y virtual en América Latina. Salta, Argentina: Ediciones Universidad Católica de Salta.

Reyes, J. (2013). Transactional Distance Theory Is It Here to Stay? Distance Learning. 10(3):43-50.

Santos-Junior, V. \& Monteiro, J. (2020). Educação e COVID-19: as tecnologias digitais mediando a aprendizagem em tempos de pandemia. Revista Encantar - Educação, Cultura e Sociedade. 2, janeiro-dezembro, 01-15. Disponível em https://www.revistas.uneb.br/index.php/encantar/article/ view/8583

Simonson, M., Smaldino, S. \& Zvacek, S. (2017). Teaching and Learning at a Distance. Foundations of Distance Education. North Carolina, USA: Information Age Publishing.

Wedemeyer, C. (1981). Learning at the Back Door, Reflections on NonTraditional Learning in the Lifespan. Winsconsin, USA: The University of Winsconsin Press.

Zawacki-Richter, O. (2019). The Industrialization Theory of Distance Education Revisited. In Jung, I. (ed.). Open and Distance Education Theory Revisited. Implications for the Digital Era, pp. 21-19. Gateway East, Singapore: Springer. 